\title{
Evolution during refrigerated storage of bioactive compounds and quality characteristics of grapefruit [Citrus paradisi (Macf.)] juice treated with UV-C light
}

\author{
Enzo L.M. La Cava*, Sonia C. Sgroppo \\ Laboratorio de Tecnología Química y Bromatología, Facultad de Ciencias Exactas, Naturales y Agrimensura, UNNE, Av. Libertad 5460, \\ Corrientes 3400, Argentina
}

\section{A R T I C L E I N F O}

\section{Article history:}

Received 14 July 2014

Received in revised form

22 March 2015

Accepted 5 April 2015

Available online 14 April 2015

\section{Keywords:}

Non-thermal technology

Organic acids

Naringin

Antioxidant capacity

Microbiological quality

\begin{abstract}
A B S T R A C T
The effect of the UV-C light (doses: $0.0-3.94 \mathrm{~J} / \mathrm{cm}^{2}$ ) on the main bioactive compounds of grapefruit juice and their stability were evaluated throughout 30 and 16 days of storage at 4 and $10{ }^{\circ} \mathrm{C}$ respectively. Organic acids (citric, malic, ascorbic and tartaric) and flavonoids (naringin, hesperidin and neohesperidin) were quantified by HPLC, whereas total phenols and the antioxidant capacity were determined by spectrophotometric methods. The UV-C treatments caused a significant decrease (15\%-30\%) in ascorbic acid and antioxidant capacity $(10 \%-27 \%)$, which was related to the applied dose. However, no changes $(\mathrm{p}>0.05)$ in others organics acids, individual flavonoids, total phenols, $\mathrm{pH}$, ${ }^{\circ}$ Brix, color and titratable acidity were observed after UV-C treatment. During the storage at both temperatures, a decrease in the neohesperidin levels (43\%-53\%) was detected whereas the others parameters analyzed did not show changes $(\mathrm{p}>0.05)$. The microbiological quality of grapefruit juices treated with $3.94 \mathrm{~J} / \mathrm{cm}^{2}$ was maintained for 15 and 10 days at 4 and $10{ }^{\circ} \mathrm{C}$ respectively.
\end{abstract}

(c) 2015 Elsevier Ltd. All rights reserved.

\section{Introduction}

The grapefruit [Citrus paradisi (Macf.)] juices are produced by industries all over the world due to the preference of the consumer based on its taste. Furthermore, they have high nutritional values and health-promoting compounds, being the ascorbic acid one of the most important. Ascorbic acid is the main compound with vitamin $C$ activity and is a natural antioxidant that may inhibit the development of major oxidative human reactions. This compound, together with citric, malic and tartaric acid contribute to flavor attributes and are used as "fingerprints" to detect the quality of the juice (Cen, Bao, He, \& Sun, 2007). Other bioactive compounds present in the grapefruit juice are the flavonoids, which are associated with biological properties, including antioxidant activity, drug interactions (de Castro, Mertens-Talcott, Derendorf, \& Butterweck, 2007), anti-inflammatory and anti-tumor effects (Fujita et al., 2008; Kim et al., 2008). The naringin is the main flavonoid in grapefruit juice and it is responsible for its bitter taste. Other neohesperidosides are present in fewer amounts, such as

\footnotetext{
* Corresponding author. Fax: +54 3794473930.

E-mail address: enzolacava@hotmail.com (E.L.M. La Cava).
}

neohesperidin, hesperidin, poncirin and neoeriocitrin (Igual, García-Martínez, Camacho, \& Martínez-Navarrete, 2011). Currently, there is a strong demand for technologies ensuring the stability of the bioactive compounds in foods (Lopez-Rubio, Gavara, \& Lagaron, 2006).

Traditionally, fruit juices have been pasteurized by heat treatment in order to prolong their shelf life. However, this treatment may cause irreversible losses of nutritional quality and antioxidant activity in the juice, thereby adversely affecting their properties health-related. On the other hand, non-thermal technologies for food processing are receiving great attention due to the ability to improve the quality and safety of foods. The UV-C light was suggested as one of the non-thermal technologies capable of ensure the microbial safety of fruit juices retaining their nutritional properties (Falguera, Garza, Pagán, Garvín, \& Ibarz, 2013; Uysal Pala \& Kirca Toklucu, 2011). The scientific criteria accepted for pasteurization of juices through a non-thermal technology UV-C is a 5 log reduction of the microorganism target (NACMCF, 2006). Moreover, the process requires very little energy compared to thermal pasteurization, also remove any traces of pesticides and it is not harmful for workers and the environment (Guerrero-Beltran \& Barbosa-Canovas, 2005; Koutchma, Forney, \& Moraru, 2009). The 
radiant energy emitted at $254 \mathrm{~nm}(112.8 \mathrm{kcal} / \mathrm{E})$ could affect the $\mathrm{O}-\mathrm{H}, \mathrm{C}-\mathrm{C}, \mathrm{C}-\mathrm{H}, \mathrm{C}-\mathrm{N}, \mathrm{H}-\mathrm{N}$ and $\mathrm{S}-\mathrm{S}$ bonds if it is absorbed. Additionally, this energy induces the crosslinking of neighboring pyrimidine nucleoside bases in the same DNA strand, blocking DNA transcription and replication and eventually causing the cell death (Guerrero-Beltran \& Barbosa-Canovas, 2005).

Although, the effect of UV-C light on the main quality characteristics have been reported in juices of orange (Tran \& Farid, 2004), apple (Noci et al., 2008), pomegranate (Uysal Pala \& Kirca Toklucu, 2011), starfruit (Bhat, Ameran, Ching Voon, Karim, \& Min Tze, 2011) and grape (Falguera et al., 2013), no works has been carried out to study the effects of the UV-C light on the organic acids, flavonoid contents and their changes during refrigerated storage of grapefruit juice. The aim of this work was to evaluate the effects of UV-C light on the levels of citric, ascorbic, malic and tartaric acids, as well as naringin, neohesperidin and hesperidin of grapefruit juice. Moreover, the evolution of these compounds during storage at 4 and $10{ }^{\circ} \mathrm{C}$ were studied. Additionally, microbial growth, $\mathrm{pH},{ }^{\circ} \mathrm{Brix}$, titratable acidity, color changes, total phenols, and antioxidant capacity were analyzed.

\section{Materials and methods}

\subsection{Preparation of juice}

The grapefruits [Citrus paradisi (Macf.)] cv 'Duncan', with uniform coloration of skin, free of cuts, similar weight and size, ratio $=5.5$, were provided by the Estación Experimental INTA Bella Vista (Corrientes, Argentina, $-28^{\circ} 30^{\prime} 52.43^{\prime \prime} \mathrm{N},-59^{\circ} 1^{\prime} 47.94^{\prime \prime} \mathrm{S}$ ). The fruits were washed with tap water, sanitized (HClO, 200 ppm/ $5 \mathrm{~min}$ ), rinsed and squeezed with a domestic extractor. The juice was filtered through a sieve (mesh aperture of 3-4 $\mathrm{mm}$ ) before the treatments.

\subsection{UV-C treatments and storage conditions}

The UV-C treatments were carried out in a chamber size of $150 \mathrm{~cm} \times 100 \mathrm{~cm} \times 60 \mathrm{~cm}$ stainless steel construction, equipped with three UV-C germicidal lamps (254 nm, UV, TUV 36W/G 36 T8 Phillips), mercury low pressure (Fig. 1). The UV radiation intensity average reached to the sample surface was quantified by chemical actinometry using an iodide/iodate solution in an area equivalent to the treatment surface (Rahn, 1997). The incident photons were calculated by assuming that, being the mixture optically opaque below $290 \mathrm{~nm}$, all of the incident photons were absorbed by the solution. In each experience, a volume of $200 \mathrm{~mL}$ of fresh grapefruit juice was placed in a container Pyrex $(27 \mathrm{~cm} \times 11 \mathrm{~cm})$ forming a film thickness of 5-7 mm under magnetic stirring (Precytec modelo AE-29, Argentina). The excess of heat generated inside the

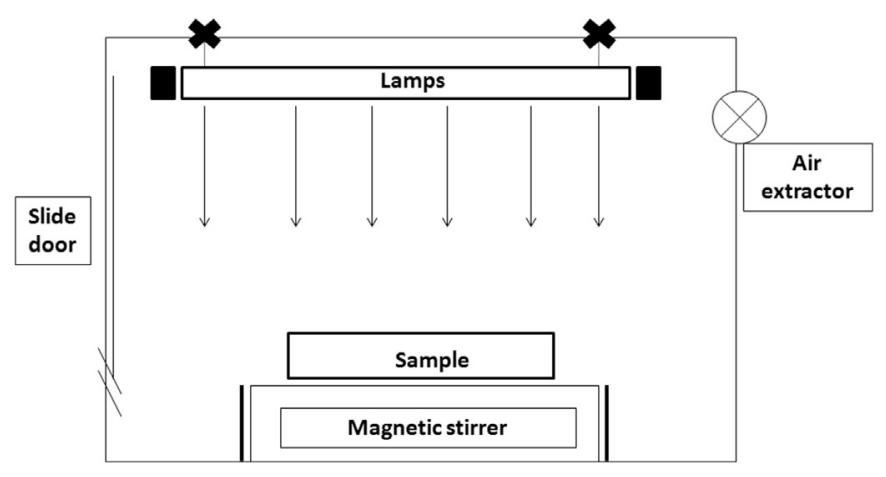

Fig. 1. Diagram of UV-C chamber (not scaled). chamber was dissipated with a fan, controlling the temperature never exceeded $25 \pm 1{ }^{\circ} \mathrm{C}$. The distance between the surface of grapefruit juice and the lamps was $17 \mathrm{~cm}$. Doses of 0.0, 1.83, 2.84 and $3.94 \mathrm{~J} / \mathrm{cm}^{2}$ were applied to grapefruit juices during the experiences. Previously, we determined that higher doses than $1.83 \mathrm{~J} /$ $\mathrm{cm}^{2}$ were effective to decrease more than 5 cycles $\log \mathrm{cfu} / \mathrm{mL}$ of Escherichia coli ATCC 25922 (data not published), close to those suggested to pasteurize orange juice by Oteiza, Giannuzzi, and Zaritzky (2010).

After the irradiation process, the samples were placed in sanitized conical containers of polypropylene $(50 \mathrm{~mL})$ with screw cap and stored as follows: at $4{ }^{\circ} \mathrm{C}$ three tubes were taken randomly without replacement for each dose at days $0,5,10,15,20,25$ and 30. At $10^{\circ} \mathrm{C}$ three tubes were taken randomly without replacement for each dose at days $0,4,8,12$ and 16 . The whole experience was performed at least 2 times.

\subsection{Content of organic acids}

The determination of tartaric, malic, ascorbic and citric acid was carried out by the method of Scherer et al. (2012). The organic acids contents were quantified by high performance liquid chromatography (Shimadzu LC-10A, Tokyo, Japan) coupled with Hypersil ODS $\mathrm{C}_{18}(250 \mathrm{~mm} \times 4.6 \mathrm{~mm}, 5 \mu \mathrm{m}$ particle size, Thermo Scientific, Whatman, MA, USA) column and the UV-visible diode array detector (Shimadzu, SPD-M20A, Tokyo, Japan) fixed at $210 \mathrm{~nm}$ for tartaric, malic and citric acid and $254 \mathrm{~nm}$ for ascorbic acid. The mobile phase was $0.01 \mathrm{~mol} / \mathrm{L} \mathrm{KH}_{2} \mathrm{PO}_{4}$ buffer solution $(\mathrm{pH}=2.60$ adjusted with o-phosphoric acid), with a flow rate of $1.0 \mathrm{~mL} / \mathrm{min}$. The samples were prepared with $5 \mathrm{~mL}$ of grapefruit juice mixed with equal parts of mobile phase and filtered through a $0.45 \mu \mathrm{m}$ nylon membrane previously to injection of $20 \mu \mathrm{L}$. The results were expressed as $\mathrm{mg} / 100 \mathrm{~mL}$ grapefruit juice based on the standard curve prepared with patterns of each acid in a range of $20-40 \mathrm{mg} /$ $100 \mathrm{~mL}$ (Sigma-Aldrich, St. Louis, MO, USA).

\subsection{Separation and quantification of flavonoids}

Five $\mathrm{mL}$ of grapefruit juice and $5.0 \mathrm{~mL}$ of a solution of ammonium oxalate $0.025 \mathrm{~mol} / \mathrm{L}$ were mixed in a tube, $5 \mathrm{~mL}$ of dimethylformamide was added, stirred and finally $\mathrm{H}_{2} \mathrm{O}$ was added to fill up $25 \mathrm{~mL}$. Subsequently the mixture was heated for $10 \mathrm{~min}$ at $90^{\circ} \mathrm{C}$, and an aliquot filtered through a membrane filter after cooling. Twenty $\mu \mathrm{L}$ of this solution was injected into the high performance liquid chromatograph (Shimadzu LC-10A, Tokyo, Japan) coupled with Hypersil ODS $C_{18}(250 \mathrm{~mm} \times 4.6 \mathrm{~mm}, 5 \mu \mathrm{m}$ particle size, Thermo Scientific, Whatman, MA, USA) column and the UV-visible-diode array (Shimadzu, SPD-M20A, Tokyo, Japan) detector fixed at $280 \mathrm{~nm}$ for naringin, hesperidin and neohesperidin. The mobile phase of acetonitrile: water: acetic acid (20:79.5:0.5) with a flow rate of $1.2 \mathrm{~mL} / \mathrm{min}$. The results were expressed as $\mathrm{mg} / 100 \mathrm{~mL}$ of grapefruit juice using standard curves prepared with patterns of each flavonoid (Sigma-Aldrich, St. Louis, MO, USA) in a solution of dimethylformamide: $0.01 \mathrm{M}$ acetic acid (20:80).

\subsection{Main physicochemical parameters}

The grapefruit juice UV absorptivity was determined at $254 \mathrm{~nm}$ (Metrolab 1700 UV-VIS) according to Oteiza et al. (2010) and turbidity with a Triton Turbidimeter (Parsen Company, Buenos Aires, Argentina). The soluble solids ( $\left.{ }^{\circ} \mathrm{Brix}\right)$ and $\mathrm{pH}$ were measured at $25^{\circ} \mathrm{C}$ using a refractometer (Model Ref 107 HandHeld, China) and a pH-meter (Metrohm meter $\mathrm{pH}$-/ion, Switzerland). The titratable acidity was determined potentiometrically with $0.1 \mathrm{~N} \mathrm{NaOH}$ and expressed as g of citric acid/100 mL of grapefruit juice. 


\subsection{Color}

The color of the fresh and treated grapefruit juice was measured with a colorimeter Minolta CR-400 Chroma Meter (Konica Minolta Sensing, Inc., Osaka, Japan). The $\mathrm{L}^{*}, \mathrm{a}^{*}, \mathrm{~b}^{*}$ parameters were measured and $\Delta \mathrm{E}^{*}$ was calculated by $\left(\left(L^{*}{ }_{0}-L^{*}\right)^{2}+\left(a^{*}{ }_{0}-a^{*}\right)^{2}+\left(b^{*}{ }_{0}-b^{*}\right)^{2}\right)^{1 / 2}$, where $L^{*}{ }_{0}, a^{*}{ }_{0}$ and $b^{*}{ }_{0}$ were measured for grapefruit juice control at the beginning of the experiment.

\subsection{Total phenols and antioxidant capacity}

The total phenolic content was determined with the FolinCiocalteu reagent (Singleton \& Rossi, 1965) using $50 \mu \mathrm{L}$ of grapefruit juice. The results were expressed as mg of gallic acid equivalents (GAE) per $100 \mathrm{~mL}$ of grapefruit juice. The free radical scavenging activity of grapefruit juice was measured according to the DPPH• method suggested by Kelebek (2010).

\subsection{Microbiological analyses}

Total aerobic count was determined by using serial dilutions on plate count agar (Britania, Argentina) with a pour plate method Serial dilutions in a range of $10^{-1}$ to $10^{-6}$ of treated and control grapefruit juices were performed with sterile $0.1 \%$ peptone water. The duplicate plates were incubated at $35 \pm 2{ }^{\circ} \mathrm{C}$ for $48 \mathrm{~h}$. The count of the total yeasts and moulds with the same dilutions was carried out on yeast extract, potato dextrose agar (Britania, Argentina) at $25{ }^{\circ} \mathrm{C}$ during 5 days using the pour plate method. Results were expressed as $\log$ colony-forming units per $\mathrm{mL}(\log \mathrm{cfu} / \mathrm{mL}$ ) (AOAC, 2000). The growth rate constant $(\mu)$ was calculated using $N_{2}=N_{1}$ $\exp \left[\mu\left(t_{2}-t_{1}\right)\right]$ where $N_{1}, N_{2}$ are the $c f u / m L$ at times $t_{1}, t_{2}$ (Painter and Loveless, 1983).

\subsection{Statistical analysis}

The experiments were performed in duplicate for each condition. The result of each determination was expressed as the mean of 3 determinations. Significant differences were evaluated by ANOVA and Duncan test $(\mathrm{p}<0.05)$ using the Info-Stat Statistical Software (Cordoba-Argentina, 2009). The Pearson correlation coefficient (R) was used $(p<0.01)$ to explain the relationship between the different compounds quantified and antioxidant capacity of the grapefruit juice.

\section{Results}

\subsection{Organic acids}

The predominant acid in grapefruit juice is the citric acid, whose values were in the range of $1584 \pm 20 \mathrm{mg} / 100 \mathrm{~mL}$ to $1759 \pm 2 \mathrm{mg} /$ $100 \mathrm{~mL}$. The malic acid content was between $37.4 \pm 0.2 \mathrm{mg} / 100 \mathrm{~mL}$ and $42.7 \pm 1.2 \mathrm{mg} / 100 \mathrm{~mL}$, whereas the tartaric acid content was between $12.0 \pm 0.4 \mathrm{mg} / 100 \mathrm{~mL}$ and $48.9 \pm 3.2 \mathrm{mg} / 100 \mathrm{~mL}$ (Tables 1 and 2). Similar contents were reported for others varieties of grapefruits (Igual, García-Martínez, Camacho, \& MartínezNavarrete, 2010; Uckoo et al., 2013).

After UV-C treatment, citric and malic acid levels were unchanged $(p<0.05)$ as was observed in orange juice treated in a range of 12.03-48.12 kJ/L (Uysal Pala \& Kirca Toklucu, 2013). The citric acid levels of control and grapefruit juice treated with $1.83 \mathrm{~J} /$ $\mathrm{cm}^{2}$ showed losses of $5-7 \%(\mathrm{p}>0.05)$ at the end of storage at $4{ }^{\circ} \mathrm{C}$, whereas the juices treated with doses higher than $2.84 \mathrm{~J} / \mathrm{cm}^{2}$ remained unchanged (Table 1 ). On the other hand, the malic acid content decreased between $14 \%$ and $20 \%$ for control and UV-C treated grapefruit juice $\left(1.83\right.$ and $2.84 \mathrm{~J} / \mathrm{cm}^{2}$ ) at 30 days. Meanwhile, in grapefruit juice treated with $3.94 \mathrm{~J} / \mathrm{cm}^{2}$ losses lower than $4 \%$ were detected (Table 1 ). The tartaric acid content was unchanged during storage at $4{ }^{\circ} \mathrm{C}$.

The initial ascorbic acid content in grapefruit juice cv. 'Duncan' was between $41.0 \pm 0.6 \mathrm{mg} / 100 \mathrm{~mL}$ and $56.9 \pm 0.6 \mathrm{mg} / 100 \mathrm{~mL}$, in the order of those reported by Uckoo et al. (2013) and Igual et al. (2010). The ascorbic acid content was significantly reduced by UV-C treatment $(\mathrm{p}<0.05)$, being the losses between 12 and $17 \%$, $20-29 \%$ and $25-35 \%$ after the application of $1.83,2.84,3.94 \mathrm{~J} / \mathrm{cm}^{2}$ respectively (Tables 1 and 2). However, in orange juices treated under continuous system, the ascorbic acid decreased more than $9 \%$ (Uysal Pala \& Kirca Toklucu, 2013; Tran \& Farid, 2004), whereas in grape juice it was more noticeable (30\%) (Falguera et al., 2013). Tikekar, Anantheswaran, Elias, and LaBorde (2011) suggested that the mechanism for UV-induced ascorbic acid degradation in juices is similar to the general mechanism for metal-catalyzed oxidation. Moreover, the decrease in the ascorbic acid content could be related to the coincidence between its absorption maximum and the peak of emission of UV-C lamps. The ascorbic acid content in untreated grapefruit juice remained without changes $(p>0.05)$ during the storage at $4{ }^{\circ} \mathrm{C}$. Meanwhile, all samples treated with UV-C did not show statistically significant changes $(\mathrm{p}>0.05)$ during the first 20 days of storage at $4{ }^{\circ} \mathrm{C}$, then, a gradual decrease was observed, with losses between a $9-14 \%$ at day $30(\mathrm{p}<0.05)$ (Table 1$)$. At $10^{\circ} \mathrm{C}$ the organic acids levels of treated and control juices remain unchanged during the 16 days of storage (Table 2).

\subsection{Flavonoids}

Flavanones constitute the $98 \%$ of the total flavonoids present in grapefruits being well known for several health promoting properties. The naringin was the main flavonoid found in grapefruit juice and values were between $17.5 \pm 0.6 \mathrm{mg} / 100 \mathrm{~mL}$ and $27.8 \pm 1.7 \mathrm{mg} / 100 \mathrm{~mL}$. The neohesperidin values were between $1.3 \pm 0.3 \mathrm{mg} / 100 \mathrm{~mL}$ and $2.5 \pm 0.6 \mathrm{mg} / 100 \mathrm{~mL}$, whereas the hesperidin was not detected (Tables 1 and 2). These values were close to those reported by Uckoo et al. (2013) and Igual et al. (2011) in other varieties of grapefruits. The naringin and neohesperidin levels in grapefruit juice remained unchanged $(p>0.05)$ after UV-C application (Tables 1 and 2).

On the other hand, the naringin content of the treated and control grapefruit juices was unchanged and showed similar behavior during storage at 4 and $10{ }^{\circ} \mathrm{C}(\mathrm{p}>0.05)$. However, the neohesperidin level showed losses of $43-58 \%$ after 15 days of storage in all grapefruit juices (Tables 1 and 2). According to our knowledge, there are no reports about the effects of UV-C treatment on individual flavonoids of grapefruit juice; however, there are several reports concerning the effects of UV-C radiation on total flavonoids of other juices. In pineapple juice treated with $7.5 \mathrm{~mJ} / \mathrm{cm}^{2}$, the total flavonoids were unchanged (Goh, Noranizan, Leong, Sew, \& Sobhi, 2012), however in starfruit juice increases were found after irradiation with doses of $2.158 \mathrm{~J} / \mathrm{m}^{2}$ (Bhat et al., 2011).

\subsection{Main physicochemical parameters}

The UV absorptivity and turbidity of grapefruit juice were of $49.47 \mathrm{~cm}^{-1}$ and 2500 NTU respectively. The value of UV absorption coefficient was close to those reported for orange and guava juice and the turbidity was between the values of apple juice (900 NTU) and orange juice (3759 NTU) (Koutchma et al. 2009). The values of $\mathrm{pH},{ }^{\circ}$ Brix, and titratable acidity are presented in Tables 3 and 4 for control and treated grapefruit juice. After the UV-C application and during refrigerated storage at both temperatures, there were no 
Table 1

Organics acids and individual flavonoids content of untreated and UV-C treated grapefruit juices during 30 days of storage at $4{ }^{\circ} \mathrm{C}$.

\begin{tabular}{|c|c|c|c|c|c|c|c|c|}
\hline \multirow[t]{2}{*}{ Treatment } & \multirow[t]{2}{*}{$\mathrm{T}\left({ }^{\circ} \mathrm{C}\right)$} & \multirow[t]{2}{*}{ Days } & \multicolumn{4}{|c|}{ Organic acids (mg/100 mL) } & \multicolumn{2}{|c|}{ Flavonoids (mg/100 mL) } \\
\hline & & & Citric & Malic & Tartaric & Ascorbic & Naringin & Neohesperidin \\
\hline \multirow[t]{7}{*}{ untreated } & 4 & 0 & 1759 A-a & $37.4 \mathrm{~A}-\mathrm{a}$ & $12.7 \mathrm{~A}-\mathrm{abc}$ & $41.0 \mathrm{~A}-\mathrm{a}$ & 17.5 A-bc & $1.51 \mathrm{~A}-\mathrm{a}$ \\
\hline & & 5 & 1725 AB-ab & $37.4 \mathrm{~A}-\mathrm{a}$ & $12.1 \mathrm{~A}-\mathrm{ab}$ & $39.9 \mathrm{~A}-\mathrm{ab}$ & 18.9 A-a & $1.12 \mathrm{~A}-\mathrm{bc}$ \\
\hline & & 10 & $1704 \mathrm{~A}-\mathrm{ab}$ & $37.2 \mathrm{~A}-\mathrm{a}$ & $12.5 \mathrm{~A}-\mathrm{abc}$ & $39.1 \mathrm{~A}-\mathrm{ab}$ & $16.6 \mathrm{~A}-\mathrm{c}$ & $1.25 \mathrm{~A}-\mathrm{ab}$ \\
\hline & & 15 & $1695 \mathrm{AB}-\mathrm{ab}$ & $37.2 \mathrm{~A}-\mathrm{a}$ & $11.6 \mathrm{AB}-\mathrm{a}$ & $36.5 \mathrm{~A}-\mathrm{b}$ & 17.0 A-bc & $0.83 \mathrm{~A}-\mathrm{c}$ \\
\hline & & 20 & $1704 \mathrm{~A}-\mathrm{ab}$ & 33.8 A-b & $12.8 \mathrm{AB}-\mathrm{abc}$ & $38.5 \mathrm{~A}-\mathrm{ab}$ & $17.4 \mathrm{~A}-\mathrm{bc}$ & $0.72 \mathrm{~A}-\mathrm{c}$ \\
\hline & & 25 & $1682 \mathrm{~A}-\mathrm{ab}$ & $31.8 \mathrm{~A}-\mathrm{bc}$ & $13.7 \mathrm{~A}-\mathrm{C}$ & $38.0 \mathrm{~A}-\mathrm{ab}$ & $17.8 \mathrm{~A}-\mathrm{abc}$ & $0.86 \mathrm{~A}-\mathrm{c}$ \\
\hline & & 30 & 1667 AB-b & $29.8 \mathrm{~A}-\mathrm{c}$ & $13.5 \mathrm{~A}-\mathrm{bc}$ & $38.0 \mathrm{~A}-\mathrm{ab}$ & $18.2 \mathrm{~A}-\mathrm{ab}$ & $0.83 \mathrm{~A}-\mathrm{c}$ \\
\hline Pooled SD & & & 40 & 1.1 & 0.8 & 0.9 & 0.7 & 0.21 \\
\hline \multirow[t]{7}{*}{$1.83 \mathrm{~J} / \mathrm{cm}^{2}$} & & 0 & $1750 \mathrm{~A}-\mathrm{ab}$ & $38.3 \mathrm{~A}-\mathrm{a}$ & $12.0 \mathrm{AB}-\mathrm{ab}$ & 33.9 B-ab & $18.3 \mathrm{~A}-\mathrm{b}$ & $1.50 \mathrm{~A}-\mathrm{a}$ \\
\hline & & 5 & $1712 \mathrm{~A}-\mathrm{c}$ & $38.5 \mathrm{~A}-\mathrm{a}$ & $12.3 \mathrm{AB}-\mathrm{ab}$ & 34.2 B-b & $19.2 \mathrm{~A}-\mathrm{a}$ & $1.13 \mathrm{~A}-\mathrm{b}$ \\
\hline & & 10 & 1798 B-b & $36.9 \mathrm{~A}-\mathrm{a}$ & $14.8 \mathrm{~A}-\mathrm{C}$ & 34.3 B-b & $18.1 \mathrm{C}-\mathrm{a}$ & $1.53 \mathrm{~A}-\mathrm{a}$ \\
\hline & & 15 & $1628 \mathrm{~B}-\mathrm{C}$ & 39.5 AB-ab & $11.4 \mathrm{~A}-\mathrm{a}$ & $33.2 \mathrm{AB}-\mathrm{ab}$ & 16.6 A-c & $0.79 \mathrm{~A}-\mathrm{c}$ \\
\hline & & 20 & 1646 B-c & 36.2 B-ab & $11.4 \mathrm{~A}-\mathrm{ab}$ & 32.1 B-ac & $16.8 \mathrm{~A}-\mathrm{c}$ & $0.66 \mathrm{~A}-\mathrm{c}$ \\
\hline & & 25 & $1638 \mathrm{~A}-\mathrm{c}$ & $34.2 \mathrm{~B}-\mathrm{ab}$ & 13.2 A-bc & $31.0 \mathrm{~B}-\mathrm{c}$ & $18.3 \mathrm{~A}-\mathrm{b}$ & $0.79 \mathrm{~A}-\mathrm{c}$ \\
\hline & & 30 & 1629 A-c & 32.6 B-b & $12.9 \mathrm{~A}-\mathrm{ab}$ & 30.4 B-c & 17.6 A-b & $0.82 \mathrm{~A}-\mathrm{c}$ \\
\hline Pooled SD & & & 46 & 1.2 & 0.9 & 1.1 & 0.4 & 0.10 \\
\hline \multirow[t]{7}{*}{$2.84 \mathrm{~J} / \mathrm{cm}^{2}$} & & 0 & 1715 A-a & $39.8 \mathrm{~A}-\mathrm{a}$ & $12.3 \mathrm{AB}-\mathrm{a}$ & 28.9 C-ab & $17.7 \mathrm{~A}-\mathrm{ab}$ & $1.63 \mathrm{~A}-\mathrm{a}$ \\
\hline & & 5 & 1764 B-b & $37.4 \mathrm{~A}-\mathrm{b}$ & $12.7 \mathrm{~B}-\mathrm{ab}$ & $28.2 \mathrm{C}-\mathrm{b}$ & $18.9 \mathrm{~A}-\mathrm{C}$ & $1.05 \mathrm{~A}-\mathrm{b}$ \\
\hline & & 10 & $1716 \mathrm{AB}-\mathrm{a}$ & $37.5 \mathrm{~A}-\mathrm{b}$ & 13.6 A-b & 29.2 C-ab & $17.5 \mathrm{BC}-\mathrm{ab}$ & $1.47 \mathrm{~A}-\mathrm{a}$ \\
\hline & & 15 & $1706 \mathrm{~A}-\mathrm{a}$ & 39.8 B-a & $12.7 \mathrm{BC}-\mathrm{ab}$ & 30.0 BC-a & $16.9 \mathrm{~A}-\mathrm{b}$ & 0.93 A-bc \\
\hline & & 20 & 1646 B-c & $38.0 \mathrm{C}-\mathrm{ab}$ & 13.2 B-ab & 28.9 C-ab & 16.9 A-b & $0.70 \mathrm{~A}-\mathrm{c}$ \\
\hline & & 25 & 1715 A-a & $34.2 \mathrm{~B}-\mathrm{C}$ & $12.4 \mathrm{AB}-\mathrm{a}$ & 26.4 C-c & $18.0 \mathrm{~A}-\mathrm{bc}$ & $0.76 \mathrm{~A}-\mathrm{c}$ \\
\hline & & 30 & 1718 B-a & $32.7 \mathrm{~B}-\mathrm{c}$ & $12.3 \mathrm{~A}-\mathrm{a}$ & $26.4 \mathrm{C}-\mathrm{c}$ & 17.6 A-ab & $0.76 \mathrm{~A}-\mathrm{c}$ \\
\hline Pooled SD & & & 18 & 1.1 & 0.6 & 0.8 & 0.5 & 0.14 \\
\hline \multirow[t]{7}{*}{$3.94 \mathrm{~J} / \mathrm{cm}^{2}$} & & 0 & $1692 \mathrm{~A}-\mathrm{a}$ & $38.4 \mathrm{~A}-\mathrm{ab}$ & $12.3 \mathrm{~A}-\mathrm{a}$ & $26.6 \mathrm{D}-\mathrm{a}$ & $18.6 \mathrm{~A}-\mathrm{a}$ & $1.31 \mathrm{~A}-\mathrm{a}$ \\
\hline & & 5 & $1703 \mathrm{~A}-\mathrm{ab}$ & $39.5 \mathrm{~A}-\mathrm{ab}$ & $12.0 \mathrm{~A}-\mathrm{ab}$ & $27.2 \mathrm{C}-\mathrm{a}$ & $18.4 \mathrm{~A}-\mathrm{a}$ & $1.04 \mathrm{~A}-\mathrm{b}$ \\
\hline & & 10 & 1747 AB-b & $40.2 \mathrm{~A}-\mathrm{a}$ & $13.5 \mathrm{~A}-\mathrm{c}$ & 26.8 D-a & 16.9 AB-bcd & $0.75 \mathrm{~A}-\mathrm{c}$ \\
\hline & & 15 & $1673 \mathrm{AB}-\mathrm{a}$ & 40.9 B-a & 13.1 C-bc & $26.3 \mathrm{C}-\mathrm{a}$ & $16.8 \mathrm{~A}-\mathrm{cd}$ & $0.96 \mathrm{~A}-\mathrm{bc}$ \\
\hline & & 20 & $1705 \mathrm{~A}-\mathrm{ab}$ & 39.4 D-b & 13.1 B-bc & 24.7 D-b & $16.5 \mathrm{~A}-\mathrm{d}$ & $0.71 \mathrm{~A}-\mathrm{c}$ \\
\hline & & 25 & 1670 A-a & 38.1 C-bc & $11.6 \mathrm{~A}-\mathrm{a}$ & 22.9 D-c & 18.2 A-ab & $0.73 \mathrm{~A}-\mathrm{c}$ \\
\hline & & 30 & 1679 AB-a & 36.9 C-c & $12.4 \mathrm{~A}-\mathrm{abc}$ & $22.8 \mathrm{D}-\mathrm{c}$ & 18.0 A-abc & $0.74 \mathrm{~A}-\mathrm{c}$ \\
\hline Pooled SD & & & 24 & 0.8 & 0.6 & 0.7 & 0.7 & 0.15 \\
\hline
\end{tabular}

Results were presented as "means \pm standard error" $(\mathrm{n}=3)$.

Values in the same columns with different uppercase letters (A-D) indicate significant differences $(p \leq 0.05)$ between treatments for the same time of storage.

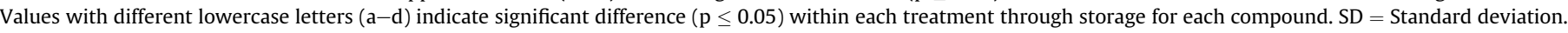

Table 2

Organics acids and individual flavonoids content of untreated and UV-C treated grapefruit juices during 16 days of storage at $10{ }^{\circ} \mathrm{C}$.

\begin{tabular}{|c|c|c|c|c|c|c|c|c|}
\hline \multirow[t]{2}{*}{ Treatment } & \multirow[t]{2}{*}{$\mathrm{T}\left({ }^{\circ} \mathrm{C}\right)$} & \multirow[t]{2}{*}{ Days } & \multicolumn{4}{|c|}{ Organic acids (mg/100 mL) } & \multicolumn{2}{|c|}{ Flavonoids (mg/100 mL) } \\
\hline & & & Citric & Malic & Tartaric & Ascorbic & Naringin & Neohesperidin \\
\hline \multirow[t]{5}{*}{ untreated } & 10 & 0 & 1628 A-a & $42.7 \mathrm{~A}-\mathrm{a}$ & 48.9 A-a & 56.9 A-a & $25.6 \mathrm{~A}-\mathrm{ab}$ & $1.60 \mathrm{~A}-\mathrm{a}$ \\
\hline & & 4 & $1591 \mathrm{~A}-\mathrm{a}$ & 37.6 A-b & $41.5 \mathrm{~A}-\mathrm{C}$ & $57.0 \mathrm{~A}-\mathrm{a}$ & $26.7 \mathrm{~A}-\mathrm{a}$ & $2.18 \mathrm{~A}-\mathrm{ab}$ \\
\hline & & 8 & 1645 AB-a & $38.8 \mathrm{~A}-\mathrm{ab}$ & $41.0 \mathrm{AB}-\mathrm{C}$ & $56.7 \mathrm{~A}-\mathrm{a}$ & $24.8 \mathrm{AB}-\mathrm{b}$ & $1.56 \mathrm{~A}-\mathrm{ab}$ \\
\hline & & 12 & $1596 \mathrm{~A}-\mathrm{a}$ & $38.9 \mathrm{~A}-\mathrm{ab}$ & $43.3 \mathrm{~A}-\mathrm{bc}$ & 56.9 A-a & $24.4 \mathrm{~A}-\mathrm{b}$ & $1.55 \mathrm{~A}-\mathrm{ab}$ \\
\hline & & 16 & $1624 \mathrm{AB}-\mathrm{a}$ & $42.0 \mathrm{~A}-\mathrm{ab}$ & $42.2 \mathrm{~A}-\mathrm{C}$ & 56.9 A-a & $26.2 \mathrm{~A}-\mathrm{ab}$ & $1.06 \mathrm{~A}-\mathrm{b}$ \\
\hline Pooled SD & & & 37 & 2.8 & 2.3 & 0.8 & 0.9 & 0.41 \\
\hline \multirow[t]{5}{*}{$1.83 \mathrm{~J} / \mathrm{cm}^{2}$} & & 0 & $1584 \mathrm{~A}-\mathrm{a}$ & $41.1 \mathrm{~A}-\mathrm{a}$ & $39.1 \mathrm{C}-\mathrm{a}$ & $49.9 \mathrm{~B}-\mathrm{ab}$ & $26.5 \mathrm{AB}-\mathrm{a}$ & $1.62 \mathrm{~A}-\mathrm{a}$ \\
\hline & & 4 & $1670 \mathrm{AB}-\mathrm{b}$ & 43.3 B-abc & $32.4 \mathrm{~B}-\mathrm{C}$ & $50.8 \mathrm{~B}-\mathrm{abc}$ & $26.5 \mathrm{~A}-\mathrm{ab}$ & $1.75 \mathrm{~A}-\mathrm{a}$ \\
\hline & & 8 & 1625 A-ab & 41.8 AB-ab & $40.6 \mathrm{AB}-\mathrm{a}$ & 49.3 B-b & $25.0 \mathrm{AB}-\mathrm{b}$ & $1.98 \mathrm{~A}-\mathrm{a}$ \\
\hline & & 12 & 1664 BC-b & $41.5 \mathrm{AB}-\mathrm{a}$ & 45.9 A-b & $52.3 \mathrm{~B}-\mathrm{ac}$ & $24.4 \mathrm{~A}-\mathrm{ab}$ & $1.57 \mathrm{~A}-\mathrm{ab}$ \\
\hline & & 16 & $1508 \mathrm{~A}-\mathrm{a}$ & 44.4 BC-bc & 39.9 A-a & $51.8 \mathrm{~B}-\mathrm{ac}$ & $25.8 \mathrm{~A}-\mathrm{ab}$ & $1.03 \mathrm{~A}-\mathrm{b}$ \\
\hline Pooled SD & & & 36 & 1.6 & 1.8 & 1.4 & 0.7 & 0.28 \\
\hline \multirow[t]{5}{*}{$2.84 \mathrm{~J} / \mathrm{cm}^{2}$} & & 0 & 1639 A-a & $40.1 \mathrm{~A}-\mathrm{a}$ & 39.7 BC-a & $45.6 \mathrm{C}-\mathrm{a}$ & $27.6 \mathrm{~A}-\mathrm{a}$ & $2.15 \mathrm{~A}-\mathrm{ab}$ \\
\hline & & 4 & $1731 \mathrm{~B}-\mathrm{c}$ & 45.7 B-b & 34.8 B-b & $48.2 \mathrm{C}-\mathrm{a}$ & $25.4 \mathrm{~A}-\mathrm{c}$ & $2.42 \mathrm{~A}-\mathrm{a}$ \\
\hline & & 8 & 1704 B-bc & 44.6 B-b & $36.0 \mathrm{~A}-\mathrm{ab}$ & $47.2 \mathrm{~B}-\mathrm{a}$ & $23.4 \mathrm{~B}-\mathrm{d}$ & $1.90 \mathrm{~A}-\mathrm{bc}$ \\
\hline & & 12 & 1694 C-bc & 45.9 B-b & 37.5 B-ab & $47.8 \mathrm{C}-\mathrm{a}$ & $25.5 \mathrm{AB}-\mathrm{C}$ & $1.47 \mathrm{~A}-\mathrm{c}$ \\
\hline & & 16 & 1665 B-ab & $46.0 \mathrm{C}-\mathrm{b}$ & 34.7 A-b & $45.9 \mathrm{C}-\mathrm{a}$ & $26.5 \mathrm{AB}-\mathrm{b}$ & $0.90 \mathrm{~A}-\mathrm{d}$ \\
\hline Pooled SD & & & 30 & 1.1 & 2.2 & 1.7 & 0.4 & 0.32 \\
\hline \multirow[t]{5}{*}{$3.94 \mathrm{~J} / \mathrm{cm}^{2}$} & & 0 & 1615 A-a & $42.6 \mathrm{~A}-\mathrm{a}$ & $45.1 \mathrm{AB}-\mathrm{a}$ & $42.1 \mathrm{D}-\mathrm{a}$ & $27.7 \mathrm{~A}-\mathrm{a}$ & $2.13 \mathrm{~A}-\mathrm{a}$ \\
\hline & & 4 & 1597 A-a & 46.2 B-a & $42.1 \mathrm{~A}-\mathrm{ab}$ & $44.3 \mathrm{D}-\mathrm{a}$ & 28.0 B-b & $1.8 \mathrm{~A}-\mathrm{ab}$ \\
\hline & & 8 & 1607 A-a & 43.5 B-a & 42.5 B-a & $44.2 \mathrm{C}-\mathrm{a}$ & $26.3 \mathrm{~A}-\mathrm{ab}$ & $1.63 \mathrm{~A}-\mathrm{ab}$ \\
\hline & & 12 & 1607 AB-a & $42.9 \mathrm{AB}-\mathrm{a}$ & $44.3 \mathrm{~A}-\mathrm{a}$ & $43.4 \mathrm{D}-\mathrm{a}$ & 25.9 B-b & $1.07 \mathrm{~A}-\mathrm{b}$ \\
\hline & & 16 & 1639 AB-a & $42.8 \mathrm{AB}-\mathrm{a}$ & 39.2 A-b & 45.9 C-a & $27.2 \mathrm{~B}-\mathrm{ab}$ & $0.80 \mathrm{~A}-\mathrm{c}$ \\
\hline Pooled SD & & & 36 & 1.5 & 1.4 & 1.4 & 1.0 & 0.34 \\
\hline
\end{tabular}

Results were presented as "means + standard error" $(\mathrm{n}=3)$.

Values in the same columns with different uppercase letters $(A-D)$ indicate significant differences $(p \leq 0.05)$ between treatments for the same time of storage.

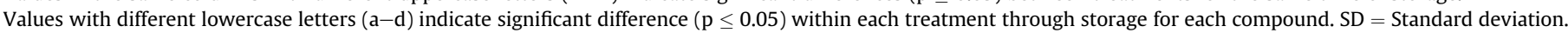


Table 3

Main physicochemical parameters quality, $\Delta \mathrm{E}^{*}$, total phenols and EC $50 \%$ of untreated and UV-C treated grapefruit juices during 30 days of storage at $4{ }^{\circ} \mathrm{C}$.

\begin{tabular}{|c|c|c|c|c|c|c|c|c|}
\hline Treatment & $\mathrm{T}\left({ }^{\circ} \mathrm{C}\right)$ & Days & $\mathrm{pH}$ & ${ }^{\circ}$ Brix & $\begin{array}{l}\text { Titratable acitidy } \\
\text { (g citric acid/100 mL) }\end{array}$ & $\Delta \mathrm{E}^{*}$ & Total phenols (mg GAE/100 mL) & EC $50 \%\left(\mathrm{DPPH}^{\bullet}\right)$ \\
\hline \multirow[t]{7}{*}{ untreated } & 4 & 0 & $3.2 \mathrm{~A}-\mathrm{ab}$ & $9.7 \mathrm{~A}-\mathrm{a}$ & $1.6 \mathrm{~A}-\mathrm{ab}$ & -- & $73.0 \mathrm{~A}-\mathrm{ab}$ & $0.0026 \mathrm{~A}-\mathrm{ab}$ \\
\hline & & 5 & $3.1 \mathrm{~A}-\mathrm{a}$ & $9.6 \mathrm{~A}-\mathrm{ab}$ & $1.6 \mathrm{~A}-\mathrm{bc}$ & $0.3 \mathrm{~A}-\mathrm{a}$ & $79.8 \mathrm{~A}-\mathrm{c}$ & $0.0026 \mathrm{~A}-\mathrm{ab}$ \\
\hline & & 10 & $3.2 \mathrm{~A}-\mathrm{ab}$ & 9.6 A-ab & $1.6 \mathrm{~A}-\mathrm{c}$ & $0.4 \mathrm{~A}-\mathrm{b}$ & 70.7 A-bd & 0.0024 A-ab \\
\hline & & 15 & $3.1 \mathrm{~A}-\mathrm{a}$ & $9.5 \mathrm{~A}-\mathrm{b}$ & $1.5 \mathrm{AB}-\mathrm{a}$ & $0.7 \mathrm{~A}-\mathrm{b}$ & 68.9 A-d & $0.0022 \mathrm{~A}-\mathrm{a}$ \\
\hline & & 20 & $3.3 \mathrm{~A}-\mathrm{b}$ & 9.6 A-ab & $1.5 \mathrm{~A}-\mathrm{a}$ & $0.7 \mathrm{~A}-\mathrm{b}$ & $74.0 \mathrm{~A}-\mathrm{b}$ & $0.0023 \mathrm{~A}-\mathrm{ab}$ \\
\hline & & 25 & $3.2 \mathrm{~A}-\mathrm{b}$ & $9.6 \mathrm{~A}-\mathrm{ab}$ & $1.5 \mathrm{~A}-\mathrm{a}$ & $0.8 \mathrm{~A}-\mathrm{c}$ & $68.6 \mathrm{~A}-\mathrm{d}$ & $0.0027 \mathrm{~A}-\mathrm{ab}$ \\
\hline & & 30 & $3.1 \mathrm{~A}-\mathrm{a}$ & $9.6 \mathrm{~A}-\mathrm{b}$ & $1.5 \mathrm{~A}-\mathrm{a}$ & $0.1 \mathrm{~A}-\mathrm{c}$ & 63.0 AB-e & $0.0028 \mathrm{~A}-\mathrm{b}$ \\
\hline Pooled SD & & & 0.1 & 0.1 & 0.1 & 0.3 & 1.6 & $1.98 \mathrm{E}-04$ \\
\hline \multirow[t]{7}{*}{$1.83 \mathrm{~J} / \mathrm{cm}^{2}$} & & 0 & $3.2 \mathrm{~A}-\mathrm{ab}$ & $9.8 \mathrm{AB}-\mathrm{a}$ & $1.6 \mathrm{~A}-\mathrm{ab}$ & $0.4 \mathrm{~A}-\mathrm{a}$ & $69.6 \mathrm{~A}-\mathrm{a}$ & $0.0031 \mathrm{~B}-\mathrm{ab}$ \\
\hline & & 5 & 3.1 A-bc & $9.5 \mathrm{~A}-\mathrm{b}$ & $1.6 \mathrm{~A}-\mathrm{b}$ & 1.6 B-b & $76.5 \mathrm{~B}-\mathrm{b}$ & $0.0030 \mathrm{~B}-\mathrm{ab}$ \\
\hline & & 10 & $3.3 \mathrm{~A}-\mathrm{b}$ & 9.7 AB-ab & $1.6 \mathrm{~A}-\mathrm{b}$ & $1.1 \mathrm{~A}-\mathrm{b}$ & $69.5 \mathrm{~A}-\mathrm{a}$ & $0.0032 \mathrm{~B}-\mathrm{ab}$ \\
\hline & & 15 & $3.3 \mathrm{~A}-\mathrm{c}$ & 9.7 B-ab & 1.5 B-ab & 0.6 B-b & $66.0 \mathrm{AB}-\mathrm{ac}$ & 0.0031 B-ab \\
\hline & & 20 & $3.2 \mathrm{~A}-\mathrm{b}$ & 9.6 A-b & 1.5 B-a & $1.0 \mathrm{~B}-\mathrm{a}$ & 69.4 AB-a & $0.0028 \mathrm{AB}-\mathrm{a}$ \\
\hline & & 25 & $3.2 \mathrm{~A}-\mathrm{b}$ & $9.6 \mathrm{~A}-\mathrm{b}$ & $1.5 \mathrm{~B}-\mathrm{ab}$ & $1.1 \mathrm{~B}-\mathrm{C}$ & $62.6 \mathrm{~B}-\mathrm{c}$ & $0.0031 \mathrm{~A}-\mathrm{ab}$ \\
\hline & & 30 & $3.1 \mathrm{~A}-\mathrm{bc}$ & 9.7 B-ab & $1.6 \mathrm{~A}-\mathrm{ab}$ & $2.0 \mathrm{~A}-\mathrm{C}$ & $61.9 \mathrm{AB}-\mathrm{C}$ & $0.0034 \mathrm{AB}-\mathrm{b}$ \\
\hline Pooled SD & & & 0.1 & 0.1 & 0.1 & 0.3 & 2.3 & $1.95 \mathrm{E}-04$ \\
\hline \multirow[t]{7}{*}{$2.84 \mathrm{~J} / \mathrm{cm}^{2}$} & & 0 & $3.2 \mathrm{~A}-\mathrm{ab}$ & $9.9 \mathrm{AB}-\mathrm{a}$ & $1.6 \mathrm{~A}-\mathrm{a}$ & $1.0 \mathrm{C}-\mathrm{c}$ & 72.6 A-a & $0.0037 \mathrm{C}-\mathrm{a}$ \\
\hline & & 5 & $3.2 \mathrm{~A}-\mathrm{ab}$ & $9.6 \mathrm{~A}-\mathrm{c}$ & $1.6 \mathrm{~A}-\mathrm{b}$ & 1.3 B-a & $73.2 \mathrm{C}-\mathrm{ab}$ & 0.0036 C-a \\
\hline & & 10 & $3.4 \mathrm{~A}-\mathrm{b}$ & $9.6 \mathrm{~A}-\mathrm{C}$ & $1.6 \mathrm{~A}-\mathrm{b}$ & $0.4 \mathrm{~B}-\mathrm{bc}$ & $68.3 \mathrm{~A}-\mathrm{ab}$ & $0.0036 \mathrm{AB}-\mathrm{a}$ \\
\hline & & 15 & $3.4 \mathrm{~A}-\mathrm{a}$ & 9.8 B-ab & $1.5 \mathrm{AB}-\mathrm{a}$ & $0.4 \mathrm{~B}-\mathrm{a}$ & 61.3 B-bc & 0.0036 BC-a \\
\hline & & 20 & $3.2 \mathrm{~A}-\mathrm{ab}$ & 9.7 A-abc & $1.5 \mathrm{~A}-\mathrm{a}$ & 0.9 C-b & $60.8 \mathrm{BC}-\mathrm{bc}$ & $0.0034 \mathrm{AB}-\mathrm{a}$ \\
\hline & & 25 & $3.2 \mathrm{~A}-\mathrm{ab}$ & 9.7 A-abc & $1.5 \mathrm{~A}-\mathrm{a}$ & $1.1 \mathrm{~A}-\mathrm{d}$ & 59.8 B-b & $0.0037 \mathrm{AB}-\mathrm{a}$ \\
\hline & & 30 & $3.0 \mathrm{~A}-\mathrm{a}$ & $9.6 \mathrm{AB}-\mathrm{bc}$ & $1.5 \mathrm{~A}-\mathrm{a}$ & $1.8 \mathrm{~A}-\mathrm{d}$ & $62.1 \mathrm{~A}-\mathrm{bc}$ & 0.0040 BC-a \\
\hline Pooled SD & & & 0.1 & 0.1 & 0.1 & 0.2 & 2.4 & 4.79E-04 \\
\hline \multirow[t]{7}{*}{$3.94 \mathrm{~J} / \mathrm{cm}^{2}$} & & 0 & $3.2 \mathrm{~A}-\mathrm{abc}$ & 9.9 B-a & $1.6 \mathrm{~A}-\mathrm{a}$ & $0.3 \mathrm{~B}-\mathrm{a}$ & $69.0 \mathrm{~A}-\mathrm{ab}$ & $0.0042 \mathrm{D}-\mathrm{a}$ \\
\hline & & 5 & $3.1 \mathrm{~A}-\mathrm{ab}$ & $9.6 \mathrm{~A}-\mathrm{c}$ & $1.6 \mathrm{~A}-\mathrm{ab}$ & 0.9 C-b & 72.9 C-a & 0.0042 D-a \\
\hline & & 10 & $3.3 \mathrm{~A}-\mathrm{c}$ & $9.8 \mathrm{~B}-\mathrm{b}$ & $1.6 \mathrm{~A}-\mathrm{b}$ & $0.3 \mathrm{~A}-\mathrm{a}$ & $68.0 \mathrm{~A}-\mathrm{b}$ & 0.0040 B-a \\
\hline & & 15 & $3.0 \mathrm{~A}-\mathrm{a}$ & 9.7 AB-bc & $1.5 \mathrm{~A}-\mathrm{c}$ & $0.2 \mathrm{C}-\mathrm{c}$ & $61.6 \mathrm{~B}-\mathrm{c}$ & 0.0039 C-a \\
\hline & & 20 & $3.2 \mathrm{~A}-\mathrm{bc}$ & $9.6 \mathrm{~A}-\mathrm{c}$ & $1.6 \mathrm{~A}-\mathrm{a}$ & 0.6 C-b & $60.8 \mathrm{C}-\mathrm{c}$ & 0.0040 B-a \\
\hline & & 25 & $3.2 \mathrm{~A}-\mathrm{bc}$ & $9.6 \mathrm{~A}-\mathrm{c}$ & $1.6 \mathrm{~A}-\mathrm{a}$ & $0.8 \mathrm{~A}-\mathrm{d}$ & $60.1 \mathrm{~B}-\mathrm{c}$ & 0.0048 B-a \\
\hline & & 30 & $3.1 \mathrm{~A}-\mathrm{ab}$ & $9.6 \mathrm{AB}-\mathrm{C}$ & $1.5 \mathrm{~A}-\mathrm{a}$ & $0.8 \mathrm{~A}-\mathrm{d}$ & 59.9 B-C & 0.0045 C-a \\
\hline PSD & & & 0.1 & 0.1 & 0.1 & 0.3 & 2.1 & 4.79E-04 \\
\hline
\end{tabular}

Results were presented as "means \pm standard error" $(n=3)$.

Values in the same columns with different uppercase letters $(A-D)$ indicate significant differences $(p \leq 0.05)$ between treatments for the same time of storage.

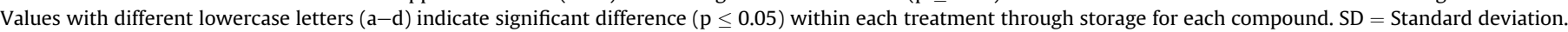

Table 4

Main physicochemical parameters quality, $\Delta \mathrm{E}^{*}$, total phenols and EC $50 \%$ of untreated and UV-C treated grapefruit juices during 16 days of storage at $10{ }^{\circ} \mathrm{C}$.

\begin{tabular}{|c|c|c|c|c|c|c|c|c|}
\hline Treatment & $\mathrm{T}\left({ }^{\circ} \mathrm{C}\right)$ & Days & $\mathrm{pH}$ & ${ }^{\circ}$ Brix & $\begin{array}{l}\text { Titratable acitidy } \\
\text { (g citric acid/100 mL) }\end{array}$ & $\Delta \mathrm{E}^{*}$ & Total phenols (mg GAE/100 mL) & EC 50\% (DPPH•) \\
\hline \multirow[t]{5}{*}{ untreated } & 10 & 0 & 2.9 A-a & $11.8 \mathrm{AB}-\mathrm{a}$ & $2.1 \mathrm{~A}-\mathrm{a}$ & -- & $86.1 \mathrm{~A}-\mathrm{a}$ & 0.0025 A-a \\
\hline & & 4 & 2.9 AB-a & $11.5 \mathrm{~A}-\mathrm{ab}$ & $2.1 \mathrm{~A}-\mathrm{a}$ & $1.1 \mathrm{~A}-\mathrm{b}$ & $84.4 \mathrm{~A}-\mathrm{a}$ & $0.0021 \mathrm{~A}-\mathrm{a}$ \\
\hline & & 8 & $2.9 \mathrm{~A}-\mathrm{a}$ & 11.6 A-ab & $2.1 \mathrm{~A}-\mathrm{a}$ & $0.8 \mathrm{~A}-\mathrm{b}$ & $85.4 \mathrm{~A}-\mathrm{a}$ & $0.0024 \mathrm{~A}-\mathrm{a}$ \\
\hline & & 12 & $2.9 \mathrm{~A}-\mathrm{a}$ & $11.3 \mathrm{~A}-\mathrm{b}$ & $2.1 \mathrm{~A}-\mathrm{a}$ & $1.1 \mathrm{~A}-\mathrm{b}$ & $74.8 \mathrm{~A}-\mathrm{b}$ & 0.0022 A-a \\
\hline & & 16 & $2.9 \mathrm{~A}-\mathrm{a}$ & $10.1 \mathrm{~A}-\mathrm{c}$ & $2.1 \mathrm{~A}-\mathrm{a}$ & $2.0 \mathrm{~A}-\mathrm{c}$ & $73.1 \mathrm{AB}-\mathrm{b}$ & $0.0021 \mathrm{~A}-\mathrm{a}$ \\
\hline PSD & & & 0.1 & 0.1 & 0.1 & 0.5 & 0.1 & 2.03E-04 \\
\hline \multirow[t]{5}{*}{$1.83 \mathrm{~J} / \mathrm{cm}^{2}$} & & 0 & 2.9 A-a & 11.5 C-ab & $2.1 \mathrm{~A}-\mathrm{a}$ & $0.7 \mathrm{~A}-\mathrm{a}$ & $86.2 \mathrm{~A}-\mathrm{a}$ & $0.0026 \mathrm{AB}-\mathrm{a}$ \\
\hline & & 4 & $2.9 \mathrm{~B}-\mathrm{a}$ & $11.6 \mathrm{~A}-\mathrm{a}$ & $2.1 \mathrm{~A}-\mathrm{a}$ & $1.1 \mathrm{~A}-\mathrm{b}$ & $85.3 \mathrm{~A}-\mathrm{a}$ & $0.0021 \mathrm{~A}-\mathrm{a}$ \\
\hline & & 8 & $2.9 \mathrm{~A}-\mathrm{a}$ & $11.5 \mathrm{~A}-\mathrm{a}$ & $2.2 \mathrm{~B}-\mathrm{b}$ & 1.0 B-b & 81.8 AB-a & 0.0026 A-a \\
\hline & & 12 & $2.9 \mathrm{~A}-\mathrm{a}$ & $11.2 \mathrm{~A}-\mathrm{bc}$ & $2.2 \mathrm{~B}-\mathrm{b}$ & $0.8 \mathrm{~B}-\mathrm{a}$ & $77.0 \mathrm{AB}-\mathrm{b}$ & $0.0022 \mathrm{~A}-\mathrm{a}$ \\
\hline & & 16 & $2.9 \mathrm{~A}-\mathrm{a}$ & $11.0 \mathrm{~B}-\mathrm{C}$ & $2.2 \mathrm{~B}-\mathrm{b}$ & $1.8 \mathrm{~B}-\mathrm{C}$ & $74.0 \mathrm{AB}-\mathrm{b}$ & $0.0023 \mathrm{AB}-\mathrm{a}$ \\
\hline PSD & & & 0.1 & 0.2 & 0.1 & 0.3 & 0.2 & $2.32 \mathrm{E}-04$ \\
\hline \multirow[t]{5}{*}{$2.84 \mathrm{~J} / \mathrm{cm}^{2}$} & & 0 & 2.9 A-a & $11.9 \mathrm{~A}-\mathrm{a}$ & $2.1 \mathrm{~A}-\mathrm{a}$ & $1.7 \mathrm{C}-\mathrm{c}$ & $86.5 \mathrm{~A}-\mathrm{a}$ & $0.0030 \mathrm{~B}-\mathrm{a}$ \\
\hline & & 4 & $3.0 \mathrm{~A}-\mathrm{a}$ & $11.8 \mathrm{~A}-\mathrm{ab}$ & $2.0 \mathrm{~A}-\mathrm{a}$ & $1.5 \mathrm{~B}-\mathrm{bc}$ & $89.3 \mathrm{~A}-\mathrm{a}$ & $0.0026 \mathrm{AB}-\mathrm{C}$ \\
\hline & & 8 & $2.9 \mathrm{~A}-\mathrm{a}$ & 11.6 A-b & $2.1 \mathrm{~A}-\mathrm{a}$ & $1.2 \mathrm{~B}-\mathrm{a}$ & 77.6 B-b & 0.0028 A-b \\
\hline & & 12 & $2.9 \mathrm{~A}-\mathrm{a}$ & $11.2 \mathrm{~A}-\mathrm{d}$ & $2.1 \mathrm{~A}-\mathrm{a}$ & $1.4 \mathrm{C}-\mathrm{b}$ & $71.4 \mathrm{~B}-\mathrm{C}$ & $0.0026 \mathrm{~A}-\mathrm{C}$ \\
\hline & & 16 & $2.9 \mathrm{~A}-\mathrm{a}$ & 11.4 C-c & $2.1 \mathrm{~A}-\mathrm{a}$ & $2.1 \mathrm{~A}-\mathrm{d}$ & $76.4 \mathrm{~B}-\mathrm{c}$ & 0.0026 BC-C \\
\hline PSD & & & 0.1 & 0.1 & 0.1 & 0.3 & 0.1 & $7.10 \mathrm{E}-05$ \\
\hline \multirow[t]{5}{*}{$3.94 \mathrm{~J} / \mathrm{cm}^{2}$} & & 0 & $2.9 \mathrm{~A}-\mathrm{a}$ & 11.7 B-a & $2.1 \mathrm{~A}-\mathrm{a}$ & $1.1 \mathrm{~B}-\mathrm{a}$ & $84.2 \mathrm{~A}-\mathrm{ab}$ & $0.0030 \mathrm{~B}-\mathrm{a}$ \\
\hline & & 4 & $3.0 \mathrm{AB}-\mathrm{a}$ & $11.6 \mathrm{~A}-\mathrm{ab}$ & $2.1 \mathrm{~A}-\mathrm{a}$ & $1.1 \mathrm{~A}-\mathrm{a}$ & 88.3 A-b & 0.0025 B-a \\
\hline & & 8 & $2.9 \mathrm{~A}-\mathrm{a}$ & 11.7 A-b & $2.1 \mathrm{~A}-\mathrm{a}$ & $1.6 \mathrm{C}-\mathrm{c}$ & 80.0 B-a & $0.0026 \mathrm{~A}-\mathrm{a}$ \\
\hline & & 12 & $2.9 \mathrm{~A}-\mathrm{a}$ & $11.2 \mathrm{~A}-\mathrm{d}$ & $2.1 \mathrm{~A}-\mathrm{a}$ & $1.4 \mathrm{C}-\mathrm{b}$ & $71.8 \mathrm{~B}-\mathrm{C}$ & $0.0024 \mathrm{~A}-\mathrm{a}$ \\
\hline & & 16 & $2.9 \mathrm{~A}-\mathrm{a}$ & 11.5 C-c & $2.1 \mathrm{~A}-\mathrm{a}$ & $2.0 \mathrm{~A}-\mathrm{d}$ & $67.4 \mathrm{~A}-\mathrm{c}$ & $0.0028 \mathrm{C}-\mathrm{a}$ \\
\hline PSD & & & 0.1 & 0.1 & 0.1 & 0.2 & 0.1 & $2.95 \mathrm{E}-04$ \\
\hline
\end{tabular}

Results were presented as "means \pm standard error" $(n=3)$.

Values in the same columns with different uppercase letters (A-D) indicate significant differences $(p<0.05)$ between treatments for the same time of storage.

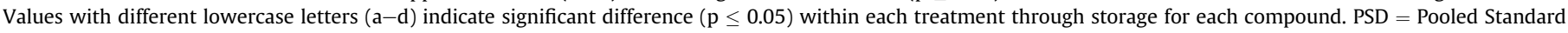
deviation. 
significant changes in those parameters $(\mathrm{p}>0.05)$ as was observed in other UV-C treated juices (Bhat et al., 2011; Caminiti et al., 2011; Falguera et al., 2013).

\subsection{Color}

The color is one most important criterion for consumer preference and it is measured as a parameter of juice quality. Immediately after UV-C treatment were detected differences lesser than 1.5 for $\Delta E^{*}$ (Tables 3 and 4 ) close to those reported by Noci et al. (2008) in apple juice UV-C treated. These differences are 'slightly noticeable' according to the classification used by Caminiti et al. (2012). A gradual trend of increased in $\Delta \mathrm{E}^{*}$ were observed during storage at both temperatures, mainly due to increases of $\mathrm{L}^{*}$, however these values did not exceed 2.5. Browning was not detected in any juice during storage.

\subsection{Total phenols and antioxidant capacity}

The total phenols content were in the range of $68.9 \pm 2.6 \mathrm{mg} /$ $100 \mathrm{~mL}$ to $86.5 \pm 3.7 \mathrm{mg} / 100 \mathrm{~mL}$, close to those reported for other varieties of grapefruit juice (Igual et al., 2010). The total phenols content after UV-C treatment did not show statistically significant changes ( $p>0.05$ ), as was observed in orange (Uysal Pala \& Kirca Toklucu, 2012), although in others fruit juices the behavior was unevenly (Falguera et al. 2013; Noci et al., 2008). Throughout the storage period, statistically significant changes $(p<0.05)$ were observed in the total phenol contents at both temperatures, which resulted in a percentage loss of the $14 \%$ for control grapefruit juices and between $11 \%$ and $20 \%$ for treated UV-C samples at the end of storage (Tables 3 and 4).

The antioxidant capacity was determined by the free radicalscavenging $\mathrm{DPPH}^{\bullet}$ reactive and values expressed as EC 50\%, being the lowest values related with a highest antioxidant activity of the compounds. The antioxidant capacity in the fresh grapefruit juice was $0.0025 \pm 7.1 \times 10^{-5} \mathrm{~mL} / \mathrm{mg}$, which was higher than those determined in other grapefruit juices (Kelebek, 2010). The antioxidant capacity showed losses of $10 \%, 22.5 \%$ and $27 \%$ after UV-C treatment with $1.83,2.84$ and $3.94 \mathrm{~J} / \mathrm{cm}^{2}$ respectively. These results are in discrepancy with those reported for orange and apple juices UV-C treated in continuo systems (Uysal Pala \& Kirca Toklucu, 2012, 2011; Noci et al., 2008). During refrigerated storage the antioxidant capacity values of control and UV-C treated grapefruit juice remained without changes $(\mathrm{p}>0.05)$ (Tables 3 and 4$)$.

\subsection{Microbial analyses}

The grapefruit juices recently squeezed had low loads of total aerobic and yeast and moulds and they were very close to the limit of detection $(<1.0 \log \mathrm{cfu} / \mathrm{mL})$. During storage at $4{ }^{\circ} \mathrm{C}$, the control

(a)
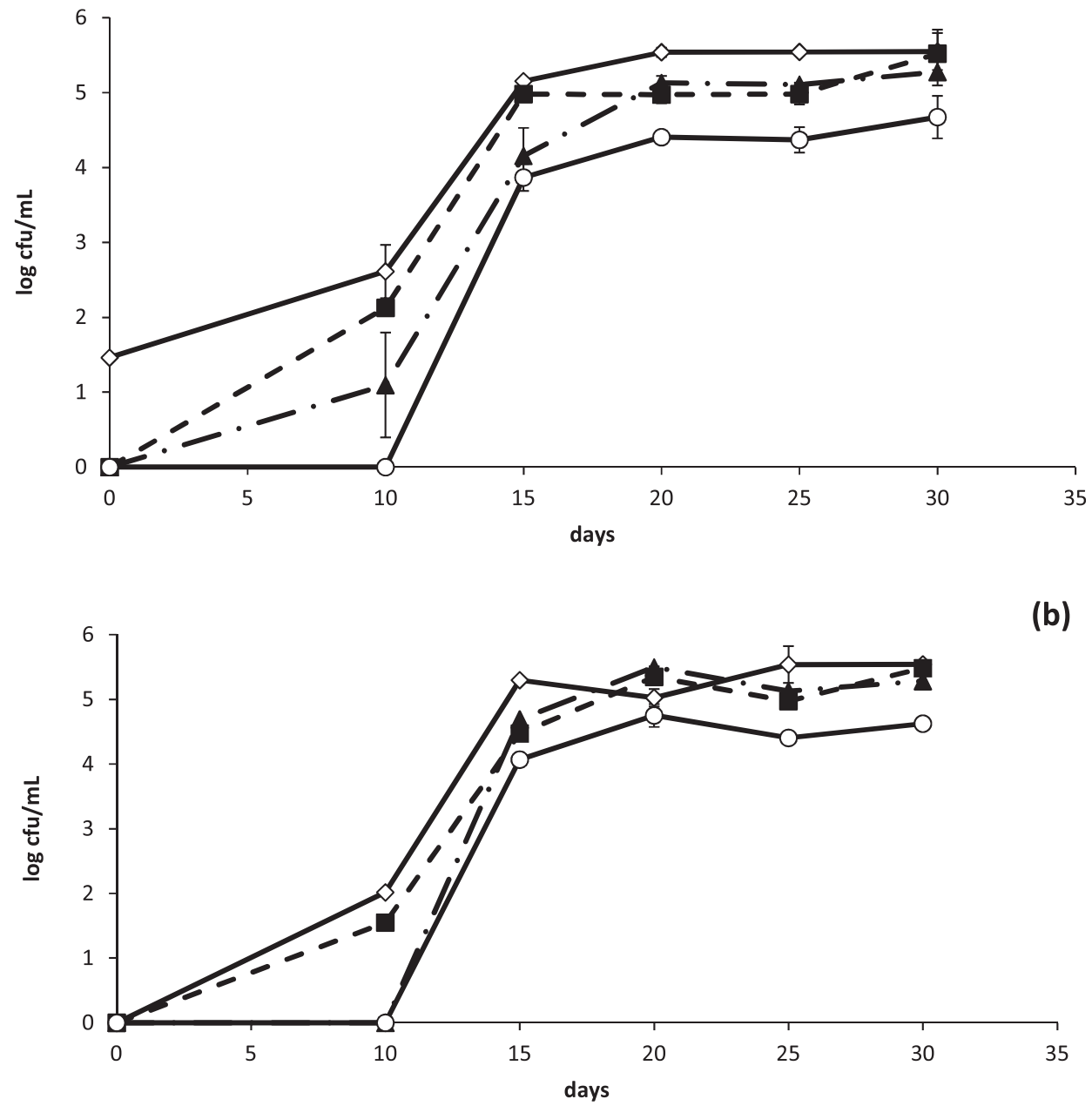

Fig. 2. Changes in total aerobic (a) and yeasts and moulds (b) counts of untreated $(\diamond)$ and UV-C treated grapefruit juices with $1.83 \mathrm{~J} / \mathrm{cm}^{2}(\boldsymbol{\square}), 2.84 \mathrm{~J} / \mathrm{cm}^{2}(\boldsymbol{\Lambda})$ and $3.94 \mathrm{~J} / \mathrm{cm}^{2}(O)$ during 30 days of storage at $4{ }^{\circ} \mathrm{C}$. 
juices showed a rapid increase in total aerobic and yeast and moulds loads ( 2.02 and $2.61 \mathrm{log} \mathrm{cfu} / \mathrm{mL}$, respectively) after 10 days and increased to $5.30 \log$ and $5.15 \log$ at day 15 , remaining unchanged until the end of storage (Fig. 2). However, the juices treated with $1.83 \mathrm{~J} / \mathrm{cm}^{2}$ showed an increase in the total aerobic and yeast and moulds counts of 1.55 and 2.12 respectively after 10 days, whereas in grapefruit juice treated with 2.84 and $3.94 \mathrm{~J} / \mathrm{cm}^{2}$ the aerobic microbial growth were not observed. At day 15 , numbers of total aerobic and yeast and moulds showed rapid growth in all grapefruit juices, being at the end of the storage the difference between the control and UV-C treated juices lesser than 1 log cfu/ $\mathrm{mL}$ (Fig. 2). During storage at $10{ }^{\circ} \mathrm{C}$, the control juice had counts of 1.32 and 1.16 in total aerobic and yeast and moulds at day 8 , after that the counts increased rapidly (3.38 and 3.08), remaining unchanged until 16 days of storage. A similar behavior was observed in grapefruit juice treated with $1.83 \mathrm{~J} / \mathrm{cm}^{2}$ with differences of less than $1 \log \mathrm{cfu} / \mathrm{mL}$ (Fig. 3). However, total aerobic and yeast and moulds count of treated grapefruit juice with 2.84 and $3.94 \mathrm{~J} / \mathrm{cm}^{2}$ were $<2 \log \mathrm{cfu} / \mathrm{mL}$ throughout 16 days storage at $10^{\circ} \mathrm{C}$. At both storage temperatures the UV-C treatments were able to retard microbial growth in the range of 10-15 days and the lowest microbial load was detected with the highest doses applied. This was in agreement with the results obtained by Uysal Pala and Kirca Toklucu (2013) and Tran and Farid (2004) in UV-C treated orange juice.

\section{Discussion}

The individual flavonoids and total phenols, as well as citric, malic and tartaric acid contents did not show changes after the UV$C$ treatment, whereas the ascorbic acid and antioxidant capacity contents decreased significantly $(\mathrm{p}<0.05)$, being more noticeable with higher UV-C doses.

In order to explain the relationships between the different compounds quantified and antioxidant capacity of the grapefruit juice, the Pearson correlation coefficient $(R)$ and $p$-values were used. The naringin content correlated highly $(R=0.95, p=0.001)$ with total phenols, which may be related to the polyphenolic structure of naringin. However, the naringin content showed not significant correlation with antioxidant capacity $(\mathrm{p}=0.220)$. Likewise, Amic, Davidovic-Amic, Beslo, and Trinajstic (2003) found that flavonoids without $3-\mathrm{OH}$ and $3^{\prime}, 4^{\prime}$ di-OH had low antioxidant capacity measured through radical scavenger DPPH$•$. Moreover, the antioxidant capacity measured through $\mathrm{DPPH} \cdot$ correlated highly $(\mathrm{p}=0.004)$ with ascorbic acid content, compound that was reported as the main antioxidant in many fruit of Citrus genus (Del Caro, Piga, Vacca, \& Agabbio, 2004).

During the storage at both temperatures, ascorbic acid content and antioxidant capacity in UV-C treated and untreated grapefruit juice remained unchanged ( $p<0.05$ ), which could be related to the insignificant headspace of the packaging and the negligible $\mathrm{O}_{2}$ -
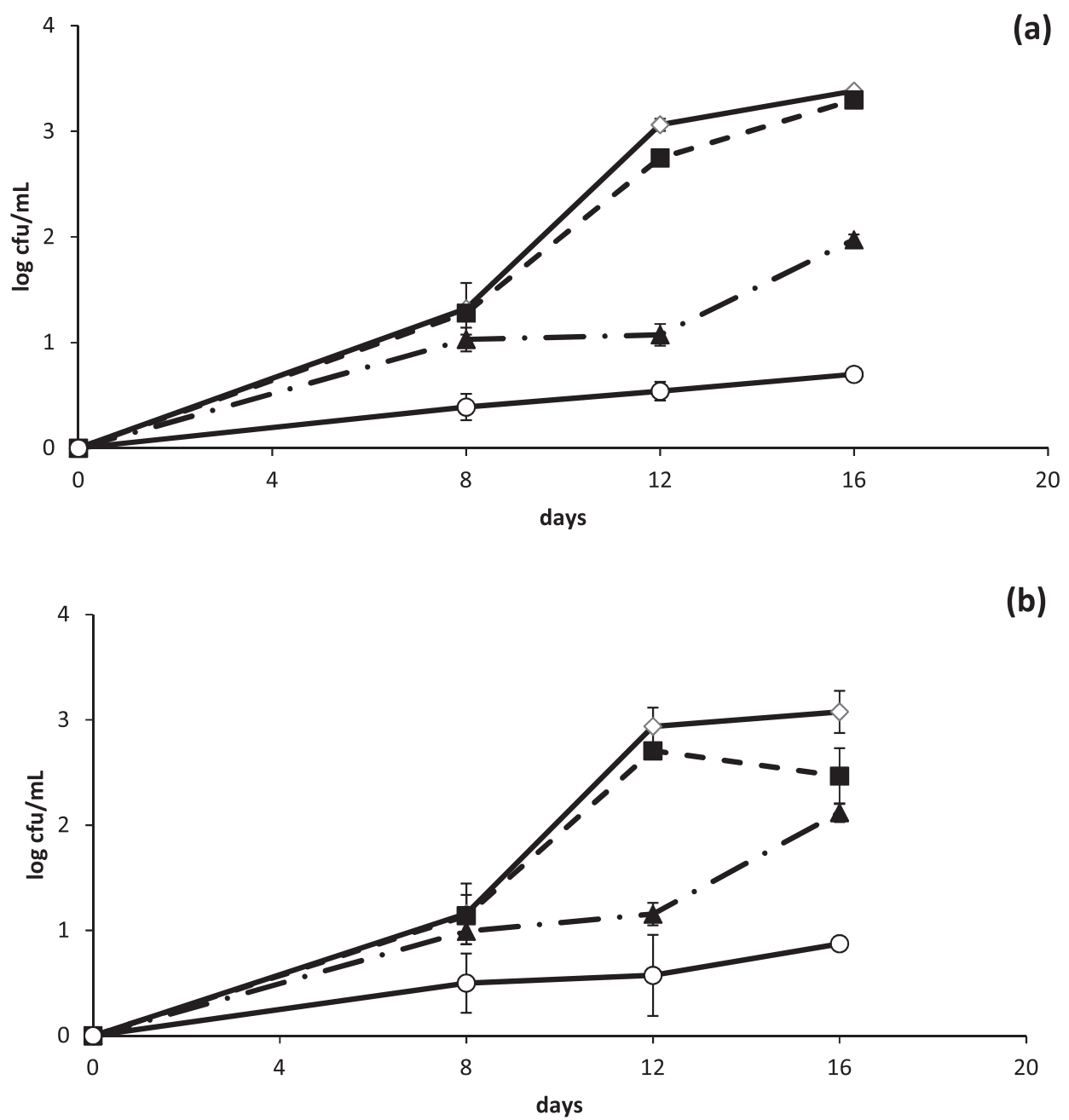

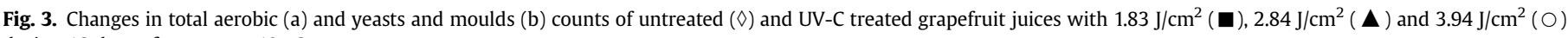
during 16 days of storage at $10^{\circ} \mathrm{C}$. 
permeability of the polypropylene tube. The other organic acids studied did not show changes in grapefruit juice treated with doses higher than $2.84 \mathrm{~J} / \mathrm{cm}^{2}$, probably due to the low load of spoilage microorganism (Chia, Rosnah, Noranizan, \& Wan Ramli, 2012). The neohesperidin content in grapefruit juice gradually decreased during storage, with notable losses after 15 days. The naringin levels, total phenols, $\mathrm{pH}$, color, ${ }^{\circ}$ Brix and titratable acidity remained unchanged $(\mathrm{p}>0.05)$.

The shelf-life of fresh citrus fruit juice is limited during storage by reduction in organoleptic quality and development of microorganisms (Tran \& Farid, 2004). In our work, may be obtained two conclusions by relating the UV-C dose applied and microbiological evolution during storage conditions. First, the total aerobic and yeast and moulds counts were lower when increasingly higher doses were applied, which could be related to the higher damage at the DNA level (Tran \& Farid, 2004). Second, in all treated grapefruit juices the microorganism growth was delayed for a longer time compared with untreated ones. Jungfer, Schwartz, and Obst (2007) reported that the delay in microbial growth is proportional to the damage received during the treatments and the type of microorganism. Supporting that, at $4{ }^{\circ} \mathrm{C}$ the growth rate constants for total aerobic were of $0.81,0.69,0.72$ and 0.62 day $^{-1}$ and for yeast and moulds were of $0.79,0.76,0.64$ and 0.59 day $^{-1}$ for doses of $0.0,1.83$, 2.84 and $3.94 \mathrm{~J} / \mathrm{cm}^{2}$ respectively. During storage at $10^{\circ} \mathrm{C}$ the growth rate constants for total aerobic were of $0.49,0.48,0.28$ and 0.10 day $^{-1}$ and for yeast and moulds were of $0.44,0.36,0.31$ and 0.13 day $^{-1}$, when treatments of $0.0,1.83,2.84$ and $3.94 \mathrm{~J} / \mathrm{cm}^{2}$ were applied. At both temperatures of storage, the samples treated with UV-C showed a decrease in the growth rate constant for total aerobic and yeast and moulds compared with untreated, and the decrease were related with the intensity of applied doses. Also it should be noted that, the presence of filamentous micro-structures in juice-air interface of samples stored was the main alteration signs and they are related to the growth of moulds and was observed in treated juices after 15 and 10 days of storage at 4 and $10{ }^{\circ} \mathrm{C}$ respectively. Yeasts and moulds have more resistance than other bacteria probably due to DNA structure and the chemical composition of the cell wall and its thickness (Tran \& Farid, 2004). Meanwhile, Uysal Pala and Kirca Toklucu (2013) found similar behavior at 4 and $10{ }^{\circ} \mathrm{C}$ on microbial growth, reported that differentiations of physicochemical characteristics of fruit juices including $\mathrm{pH}$, soluble solids and phenolic compounds may have had significant effects on microbial growth during storage in addition to the effects of storage temperature. Also, Ahmed, Chandan, Mukund, Sumeet, and Chidambaram (2014) reported that the orange juices with more citric acid content showed lower microbial load. This was in agreement with Bizri and Wahem (1994) who found differences as high as 2 logarithmic cycles in the total aerobic counts in tomato juice with different $\mathrm{pH}$ values (less than 0.4 ).

\section{Conclusion}

The UV-C treatments decreased ascorbic acid and antioxidant capacity of grapefruit juice and the effect was more noticeable when higher doses were applied. However, the naringin, neohesperidin, citric, malic, tartaric acid as well as, $\mathrm{pH}$, ${ }^{\circ}$ Brix, titratable acidity, color and total phenols were not affected.

During the refrigerated storage, the treatments with UV-C enhanced the shelf life of juices for 15 and 10 days at 4 and $10{ }^{\circ} \mathrm{C}$ respectively, due to the microbiological control achieved. The treatments were not effective to prevent loss of neohesperidin and total phenols during storage at both temperatures, while organic acids had a lower degradation in treated grapefruit juice.

Also, the naringin and ascorbic acid contents, as well as antioxidant capacity, $\mathrm{pH},{ }^{\circ}$ Brix, titratable acidity and color showed similar evolution in treated and control grapefruit juice for both storage temperatures. Then, the UV-C treatments could be suggested as a method for preservation of grapefruit juice, if they are accepted sensorially by the consumers.

\section{Acknowledgments}

This work was supported by Project PICT-2010-1496 ANPCYT (MINCYT- Argentina). The Universidad Nacional del Nordeste (Argentina) and the Consejo Nacional de Investigaciones Científicas y Técnicas (CONICET), Argentina.

\section{References}

Ahmed, H., Chandan, K. G., Mukund, M., Sumeet, S. O., \& Chidambaram, R. (2014) Effect of citric and malic acid on shelf life and sensory characteristics of orange juice. International Journal of Pharmacy and Pharmaceutical Sciences, 6.

Amic, D., Davidovic-Amic, D., Beslo, D., \& Trinajstic, N. (2003). Structure-radical scavenging activity relationship of flavonoids. Croatia Chemica Acta, 76(1), 55-61.

AOAC. (2000). Microbiological methods (17th ed.). In Official methods of analysis of the Association of Official Analytical Chemists (17th ed.), (Vol. I). AOAC International.

Bhat, R., Ameran, S. A., Ching Voon, H., Karim, A. A., \& Min Tze, L. (2011). Quality attributes of starfruit (Averrhoa carambola L.) juice treated with ultraviolet radiation. Food Chemistry, 127, 641-644.

Bizri, J. L., \& Wahem, I. A. (1994). Citric acid and antimicrobials affect microbiological stability and quality of tomato juice. Journal of Food Science, 59(1) 130-134.

Caminiti, I. M., Noci, F., Morgan, D. J., Cronin, D. A., James, G., \& Lyng, J. G. (2012). The effect of pulsed electric fields, ultraviolet light or high intensity light pulses in combination with manothermosonication on selected physico-chemical and sensory attributes of an orange and carrot juice blend. Food and Bioproducts Processing, 90, 442-448.

Caminiti, I. M., Noci, F., Muñoz, A., Whyte, P., Morgan, D. J., Cronin, D. A., et al. (2011). Impact of selected combinations of non-thermal processing technologies on the quality of an apple and cranberry juice blend. Food Chemistry, 124(4) $1387-1392$.

de Castro, W. V., Mertens-Talcott, S., Derendorf, H., \& Butterweck, V. (2007). Grapefruit juice-drug interactions: grapefruit juice and its components inhibit P-glycoprotein (ABCB1) mediated transport of talinolol in Caco-2 cells. Journal of Pharmaceutical Sciences, 96(10), 2808-2817.

Cen, H., Bao, Y., He, Y., \& Sun, D. W. (2007). Visible and near infrared spectroscopy for rapid detection of citric and tartaric acids in orange juice. Journal of Food Engineering, 82, 253-260.

Chia, S. L., Rosnah, S., Noranizan, M. A., \& Wan Ramli, W. D. (2012). The effect of storage on the quality attributes of ultraviolet-irradiated and thermally pasteurized pineapple juices. International Food Research Journal, 19(3) 1001-1010.

Del Caro, A., Piga, A., Vacca, V., \& Agabbio, M. (2004). Changes of flavonoids, vitamin $\mathrm{C}$ and antioxidant capacity in minimally processed citrus segments and juices during storage. Food Chemistry, 84, 99-105.

Falguera, V., Garza, S., Pagán, J., Garvín, A., \& Ibarz, A. (2013). Effect of UV-Vis irradiation on enzymatic activities and physicochemical properties of four grape musts from different varieties. Food Bioprocess Technology, 6 2223-2229.

Fujita, T., Kawase, A., Niwa, T., Tomohiro, N., Masuda, M., Matsuda, H., et al. (2008) Comparative evaluation of 12 immature citrus fruit extracts for the inhibition of cytochrome P450 isoform activities. Biological and Pharmaceutical Bulletin, 31(5), 925-930.

Goh, S. G., Noranizan, M., Leong, C. M., Sew, C. C., \& Sobhi, B. (2012). Effect of thermal and ultraviolet treatments on the stability of antioxidant compounds in single strength pineapple juice throughout refrigerated storage. International Food Research Journal, 19(3), 1131-1136.

Guerrero-Beltran, J. A., \& Barbosa-Canovas, G. V. (2005). Reduction of Saccharomyces cerevisiae. Escherichia coli and Listeria innocua in apple juice by ultraviolet light. Journal Food Processing Engineering, 28, 437-452.

Igual, M., García-Martínez, E., Camacho, M. M., \& Martínez-Navarrete, N. (2010) Effect of thermal treatment and storage on the stability of organic acids and the functional value of grapefruit juice. Food Chemistry, 118, 291-299.

Igual, M., García-Martínez, E., Camacho, M. M., \& Martínez-Navarrete, N. (2011). Changes in flavonoid content of grapefruit juice caused by thermal treatment and storage. Innovative Food Science and Emerging Technologies, 12, $153-162$.

Jungfer, C., Schwartz, T., \& Obst, U. (2007). UV-induced dark repair mechanisms in bacteria associated with drinking water. Water Research, 41, 188-196.

Kelebek, H. (2010). Sugars, organic acids, phenolic compositions and antioxidant activity of grapefruit (Citrus paradisi) cultivars grown in Turkey. Industrial Crops and Products, 32, 269-274. 
Kim, D. I., Lee, S. J., Lee, S. B., Park, K., Kim, W. J., \& Moon, S. K. (2008 Sep). Requirement for Ras/Raf/ERK pathway in naringin-induced G1-cell-cycle arrest via p21WAF1 expression. Carcinogenesis, 29(9), 1701-1709.

Koutchma, T., Forney, L. J., \& Moraru, C. I. (2009). UV processing effects on quality of foods. In Da-W. Sun (Ed.), Ultraviolet light in food technology: Principles and applications (pp. 53-68). USA: CRC Press, Taylor and Francis Group.

Lopez-Rubio, A., Gavara, R., \& Lagaron, J. M. (2006). Bioactive packaging: turning foods into healthier foods through biomaterials. Trends in Food Science \& Technology, 17(10), 567-575.

National Advisory Committee on Microbiological Criteria for Foods (NACMCF). (2006). Supplement requisite scientific parameters for establishing the equivalence of alternative methods of pasteurization. Journal of Food Protection, 69, 1190-1216.

Noci, F., Riener, J., Walkling-Ribeiro, M., Cronin, D. A., Morgan, D. J., \& Lyng, J. G. (2008). Ultraviolet irradiation and pulsed electric fields (PEF) in a hurdle strategy for the preservation of fresh apple juice. Journal of Food Engineering, 85(1), 141-146.

Oteiza, J. M., Giannuzzi, L., \& Zaritzky, N. (2010). Ultraviolet treatment of orange juice to inactivate Escherichia coli 0157: $\mathrm{H7}$ as affected by native microflora. Food Bioprocess Technology, 3, 603-614.

Painter, H. A., \& Loveless, J. E. (1983). Effect of temperature and pH value on the growth rate constants of nitrifying bacteria in the activated-sludge process. Water Research, 17, 237-248.

Rahn, R. (1997). Potassium iodide as a chemical actinometer for $254 \mathrm{~nm}$ Radiation: use of iodate as an electron scavenger. Photochemistry and Photobiology, 66(4), $450-455$.
Scherer, R., Rybka, A. C. P., Ballus, C. A., Meinhart, A. D., Teixeira Filho, J., \& Teixeira Godoy, H. (2012). Validation of a HPLC method for simultaneous determination of main organic acids in fruits and juices. Food Chemistry, 135, 150-154.

Singleton, V. L., \& Rossi, J. A., Jr. (1965). Colorimetry of total phenolics with phosphomolybdic-phosphotungstic acid reagents. American Journal of Enology and Viticulture, 16, 144-158.

Tikekar, R. V., Anantheswaran, R. C., Elias, R. J., \& LaBorde, L. F. (2011). Ultravioletinduced oxidation of ascorbic acid in a model juice System: Identification of degradation products. Journal of Agricultural and Food Chemistry, 59, 2844-2848.

Tran, M. T. T., \& Farid, M. (2004). Ultraviolet treatment of orange juice. Innovative Food Science \& Emerging Technologies, 5, 495-502.

Uckoo, R. M., Jayaprakasha, G. K., Somerville, J. A., Balasubramaniam, V. M., Pinarte, M., \& Patil, B. S. (2013). High pressure processing controls microbial growth and minimally alters the levels of health promoting compounds in grapefruit (Citrus paradisi Macfad) juice. Innovative Food Science and Emerging Technologies, 18, 7-14.

Uysal Pala, C., \& Kirca Toklucu, A. (2011). Effect of UV-C light on anthocyanin content and other quality parameters of pomegranate juice. Journal of Food Composition and Analysis, 24, 790-795.

Uysal Pala, C., \& Kirca Toklucu, A. (2012). Effects of UV-C light processing on some quality characteristics of grape juices. Food Bioprocess Technology, 6, $719-725$.

Uysal Pala, C., \& Kırca Toklucu, A. (2013). Microbial, physicochemical and sensory properties of UV-C processed orange juice and its microbial stability during refrigerated storage. LWT - Food Science and Technology, 50, 426-431. 